\title{
Veranstaltungsankündigung
}

\section{IK 2002}

\section{Interdisziplinäres Kolleg, Schwerpunkt: Autonomie und Emotion 1.-8. März 2002, Günne am Möhnesee}

\section{Wissenschaftliche Leitung}

Thomas Christaller

(Fraunhofer-Instituts für Autonome Intelligente Systems (AiS), Sankt Augustin)

Manfred Spitzer

(Leiter der Psychiatrischen Universitätsklinik Ulm)

Wie in den letzten Jahren findet auch 2002 in Günne am Möhnesee das Interdisziplinäre Kolleg (IK) statt. Das IK ist eine einwöchige Frühjahrsschule mit Grund- und Intensivkursen aus den Gebieten Neurobiologie, Robotik, Kognitionswissenschaft, Neuroinformatik, Philosophie und Künstliche Intelligenz - kurz gesagt, allen Disziplinen, die sich im weitesten Sinn mit Kognition und Gehirn beschäftigen. Diese Veranstaltung richtet sich an Studierende, Doktoranden/Doktorandinnen und Wissenschaftler/innen aus dem akademischen und industriellen Bereich.

Das IK ist eine intensive Veranstaltung mit einem dichten, anspruchsvollen Kursprogramm und hochkarätigen Abendvorträgen. Fast genauso wichtig ist das „Nach- und Nachtprogramm" mit Diskussionen, die oft erst im Morgengrauen am Ufer des Möhnesees enden. (Für „Alte Hasen”: Das IK ist aus den „Künstliche Intelligenz Frühjahrsschulen“" (KIFSen) hervorgegangen - gleicher Ort, gleiche Hochspannung).

Das IK bietet einen sehr breiten Einstieg in die beteiligten Disziplinen. In der ersten Wochenhälfte finden Grundkurse statt, die orientierende Einstiege für Nicht-Spezialisten in die Gebiete Neurobiologie, Kognitionswissenschaft, KI, und (Novum auf diesem IK) mathematische Modellierung komplexer Systeme bieten. In der zweiten Wochenhälfte bieten 16 Spezialkurse vertiefende Einführungen in aktuelle Forschungsthemen. Jedes IK hat ein Schwerpunktthema, dem etwa ein Viertel des Kursangebotes gewidmet ist. Beim IK 2002 lautet der Schwerpunkt „Autonomie und Emotion", und eine Reihe von Spezialkursen widmen sich diesen Aspekten - der Autonomie besonders aus Sicht der der Philosophie und der Robotik, der Emotion aus Sicht der Psychologie, Verhaltensforschung und Neurobiologie. Dies ist die persönliche Handschrift der beiden Forscher, die die inhaltliche Gesamtleitung für dieses IK übernommen haben: Prof. Dr. Thomas Christaller, Leiter des Fraunhofer-Instituts für Autonome Intelligente Systems (AiS) in Sankt Augustin, und Prof. Dr. Manfred Spitzer, Leiter der Psychiatrischen Universitätsklinik Ulm.

Ursprünglich ist das IK eine deutschsprachige Veranstaltung. Schon beim IK 2001 wurde aber die Europäisierung forciert. Die Kurse des IK 2002 werden nun erstmalig durchgängig in englischer Sprache gehalten, und gut die Hälfte der Dozenten kommt nicht aus Deutschland.

Veranstaltungssekretariat:

Christine Harms

Schloß Birlinghoven

D-53754 Sankt Augustin

Telefon: 02241-14-2473

Telefax: 02241-14-2472

Das genaue Kursangebot, die Trägerorganisationen und Sponsoren, Anmeldeformulare, Kursgebühren etc. finden sich auf den Webseiten des IK: http://www.tzi.de/ik2002/. 